\title{
HOMOMORPHIC IMAGES OF RESTRICTIVE STAR SEMIGROUPS
}

\author{
by KENNETH D. MAGILL, Jr.
}

(Received 16 January, 1969)

0. Introduction. Let $Y$ be a subspace of a topological space $X$. Then $S(X, Y)$ denotes the semigroup, under composition, of all continuous selfmaps of $X$ which also carry $Y$ into $Y$. In the special case $Y=X$, the simpler notation $S(X)$ is used. We have devoted several recent papers ( [4], [7] and [8]) to the problem of determining when $S(Z)$ and $S(X, Y)$ are isomorphic and, more generally, when $S(Z)$ is a homomorphic image of $S(X, Y)$. In this paper, we investigate the analogous problem for certain semigroups of functions on spaces which were introduced in [5]. These include semigroups of closed functions which are treated in further detail.

1. Some results on $\mathfrak{R}$-spaces. In this section, we recall some definitions and extend some results from [5, §2, pp. 526-530] which we shall subsequently need.

Definition (1.1). $\mathscr{P}(X)$ denotes the power set of a nonempty set $X$ and the subfamily of $\mathscr{P}(X)$ which consists of $X$ and all singletons of $X$ is referred to as the core of $\mathscr{P}(X)$.

Definition (1.2). Let $\mathscr{F}$ be any subfamily of $\mathscr{P}(X)$. A selfmap $f$ of $X$ is said to be $\mathscr{F}$-invariant if $f[H] \in \mathscr{F}$ for each $H \in \mathscr{F}$.

Definition (1.3). A subfamily $\mathscr{F}$ of $\mathscr{P}(X)$ is an $\Re$-family if it contains the core and each member of the family is the range of an $\mathscr{F}$-invariant function.

Definition (1.4). An $\Re$-space is a pair $(X, \mathscr{F})$ where $X$ is a nonempty set and $\mathscr{F}$ is an $\Re$-family of subsets of $X$.

We shall often take the liberty of referring to $X$ itself as an $\Re$-space. It is to be understood that some $\mathfrak{R}$-family of subsets is associated with $X$. For a discussion of $\mathfrak{R}$-families (and also families which are not $\mathfrak{R}$-families), see [5, 2 , pp. 526-530]. In particular, the following result which we state without proof is verified there.

THEOREM (1.5). Let $X$ be any nonempty set and let $\mathscr{F}$ be a family of subsets of $X$ which satisfies the following conditions:

$$
\mathscr{F} \text { contains the core of } \mathscr{P}(X) \text {, }
$$

$\mathscr{F}$ is closed under finite intersections,

Then $\mathscr{F}$ is an $\Re$-family.

The next two corollaries are immediate consequences of this theorem.

COROLLARY (1.6). The family of all closed subsets of a $T_{1}$ topological space is an $\Re$-family. 
COROLlaRY (1.7). The family of all compact subsets of a Hausdorff space, together with the space itself, is an $\mathfrak{R}$-family.

$\mathfrak{R}$-families of the type described in Theorem (1.5) play enough of a role in our considerations to make it convenient to provide them with a name.

Definition (1.8). Any subfamily of $\mathscr{P}(X)$ which satisfies conditions (1.5.1), (1.5.2) and (1.5.3) will be referred to as a principal $\mathfrak{R}$-family and the corresponding $\mathfrak{R}$-space will be referred to as a principal $\mathfrak{R}$-space.

The next result extends Theorem (2.8) of [5, p. 530]. The same basic techniques are used in the proof.

THEOREM (1.9). Let $X$ be any completely regular Hausdorff space whose cardinality does not exceed $c$, the cardinality of the continuum, and let $\mathscr{C}_{X}$ consist of the core of $\mathscr{P}(X)$ together with any family of connected subsets of $X$. Then $\mathscr{C}_{X}$ is an $\mathfrak{R}$-family.

Proof. If $X$ is totally disconnected, then $\mathscr{C}_{X}$ must necessarily be the core of $\mathscr{P}(X)$ and the conclusion is immediate. Suppose that $X$ is not totally disconnected and let $H$ be any element of $\mathscr{C}_{X}$ which is different from $X$ (it follows at once that $X$ is the range of a $\mathscr{C}_{X}$-invariant function, namely the identity function). We must prove the existence of a $\mathscr{C}_{X}$-invariant function $f$ whose range is $H$. Since $X$ is not totally disconnected, there exist two distinct points $p$ and $q$ which belong to a connected subset of $X$ and, by complete regularity, there exists a continuous function $g$ mapping $X$ into the closed unit interval $I$, such that $g(p)=0$ and $g(q)=1$. It follows that the range of $g$ is all of $I$. Now we define a selfmap of $I$. For each $x \neq 0$ in $I$, let

$$
. a_{1} a_{2} a_{3} \ldots a_{n} \ldots\left(a_{i}=0 \text { or } 1\right)
$$

denote the nonterminating binary expansion of $x$. Define the function $h$ by

$$
\begin{aligned}
& h(x)=\limsup \left\{\left(a_{1}+a_{2}+\ldots+a_{n}\right) / n\right\}, \\
& h(0)=0 .
\end{aligned}
$$

The function $h$ is discussed in [2] on page 82, where it is observed that the image of any nondegenerate subinterval (closed, open or half-open) of $I$ is all of $I$. Since the cardinality of $H$ does not exceed $c$, there exists a surjection $k$ from $I$ onto $H$. Let $f=k \circ h \circ g$ and let $K$ be any connected subset of $X$. Then $g[K]$ is a connected subset of $I$ and, consequently, must be a point or a nondegenerate interval. In the first case $h[g[K]]$ is a point and in the second $h[g[K]]=I$. It follows that $f[K]=k[h[g[K]]]$ is either a point or all of $H$. Thus $f$ is a $\mathscr{C}_{X^{-}}$invariant selfmap of $X$ whose range is $H$.

Definition (1.10). The symbol $\mathscr{C}_{\boldsymbol{X}}$ will be used exclusively for denoting an $\mathfrak{R}$-family consisting of the core of $\mathscr{P}(X)$ together with a collection of connected subsets of a completely regular Hausdorff space $X$ whose cardinality does not exceed $c$. The $\mathfrak{R}$-family consisting of the core together with all connected subsets of such a space $X$ will be denoted by $\hat{\mathscr{C}}_{X}$.

2. Dependant $\mathfrak{R}$-spaces. In the theory of topological spaces, one associates, in a very natural way, a unique topology with every subset $Y$ of a topological space $X$. The subspace 
topology on $Y$ is simply defined to be the intersections of $Y$ with the open subsets of $X$. We give an example to show that, in general, this technique does not work for $\mathfrak{R}$-spaces and then we introduce the notion of a dependant $\mathfrak{R}$-space which, in a certain sense, is analogous to a subspace of a topological space.

EXAmple (2.1). Let $X$ denote the set of natural numbers and let $A=X-\{5\}, B=$ $X-\{3,4\}$ and $Y=\{1,2,3,4,5\}$. Denote by $\mathscr{F}_{X}$ the subfamily of $\mathscr{P}(X)$ consisting of the core together with the two sets $A$ and $B$. It is not difficult to show that $\mathscr{F}_{X}$ is an $\mathfrak{R}$-family. For instance, let $f$ be any function mapping the set $\{n \in X: n \geqq 6\}$ onto $B$, such that $f(n) \geqq 6$ for $1 \leqq n \leqq 5$. Then $f[X]=f[A]=f[B]=B$ and it follows that $B$ is the range of an $\mathscr{F}_{X}$-invariant function. Similar remarks hold for $A$ and it follows that $\mathscr{F}_{X}$ is indeed an $\mathfrak{R}$-family. Actually, one can prove the more general result that the core together with any finite family of infinite subsets of any infinite set is an $\mathfrak{R}$-family [5, p. 526, Theorem (2.2)].

Now let $\mathscr{F}_{Y}$ denote the family of intersections of $Y$ with members of $\mathscr{F}_{X}$. It follows from the more general considerations of Example (2.4) in [5, p. 528] that $\mathscr{F}_{Y}$ is not an $\mathfrak{R}$-family. However, the verification in this special case is not long and it may be worthwhile to give it. $\mathscr{F}_{Y}$ consists of the core of $\mathscr{P}(Y)$ together with the two sets $A^{\prime}=\{1,2,3,4\}$ and $B^{\prime}=\{1,2,5\}$. We show that $A^{\prime}$ is not the range of any $\mathscr{F}_{Y}$-invariant function. Suppose that $f$ is $\mathscr{F}_{Y}$-invariant and $f[Y] \subset A^{\prime}$. Then $f\left[B^{\prime}\right]$ cannot be equal to $B^{\prime}$ and for cardinality reasons cannot be either $Y$ or $A^{\prime}$. Thus $f\left[B^{\prime}\right]$ must be a singleton. It follows that $f[Y]$ can consist of at most three points and therefore cannot possibly be $A^{\prime}$.

It is now apparent that one cannot always expect to get an $\Re$-family for $Y$ by intersecting $Y$ with the members of an $\mathfrak{R}$-family of $X$. There is a very natural way to provide $Y$ with an $\Re$-family which is closely related to the $\mathfrak{R}$-family $\mathscr{F}_{X}$ of $X$. One readily verifies that

$$
\mathscr{L}_{Y}=\left\{f[Y]: f[Y] \subset Y \text { and } f \text { is an } \mathscr{F}_{X} \text {-invariant selfmap of } X\right\}
$$

is indeed an $\mathfrak{R}$-family. It is appealing from the standpoint that it is the largest possible $\mathfrak{R}$-family on $Y$ whose members are images of $Y$ under $\mathscr{F}_{X}$-invariant functions which take $Y$ into $Y$. The difficulty, however, is that quite often the more interesting $\mathfrak{R}$-families are actually proper subfamilies of the latter. For consider the following

EXAMPLE (2.2). Let $X$ be any completely regular Hausdorff space with cardinality less than or equal to $c$ and let $Y$ be any nonempty subspace of $X$. Let $\mathscr{C}_{X}$, the family of all connected subsets of $X$, together with $X$ itself, be the $\Re$-family associated with $X$. It is quite natural to want to consider $\mathscr{C}_{Y}$ as a candidate for the $\mathfrak{R}$-family on $Y$. $\mathscr{C}_{Y}$, however, may well be a proper subset of $\mathscr{L}_{Y}$ defined above. To see this, let $X=[0,2]$ and let $Y=X-\{1\}$. Define a continuous selfmap of $X$ by

$$
\begin{aligned}
& f(x)=(x+1) / 2 \text { for } 0 \leqq x \leqq 1 \\
& f(x)=x \text { for } 1 \leqq x \leqq 2 .
\end{aligned}
$$

It follows that $f[Y]=[1 / 2,1) \cup(1,2]$ belongs to $\mathscr{L}_{Y}$ but certainly not to $\mathscr{C}_{Y}$. All this has led us to the following 
Definition (2.3). Let $\left(X, \mathscr{F}_{X}\right)$ and $\left(Y, \mathscr{F}_{Y}\right)$ be two $\mathfrak{R}$-spaces. We say that $\left(Y, \mathscr{F}_{Y}\right)$ is a $Y$-dependant (or simply a dependant) of $\left(X, \mathscr{F}_{X}\right)$ if $Y \subset X$ and, for each $H \in \mathscr{F}_{Y}$, there exists an $\mathscr{F}_{X}$-invariant selfmap $f$ of $X$ which satisfies the following two conditions:

The restriction of $f$ to $Y$ is an $\mathscr{F}_{Y}$-invariant selfmap of $Y$,

$$
f[Y]=H \text {. }
$$

THEOREM (2.4). Let $X$ be any completely regular Hausdorff space with cardinality less than or equal to $c$ and let $Y$ be a nonempty subspace of $X$. Then $\left(Y, \mathscr{C}_{Y}\right)$ is a dependant of $\left(X, \mathscr{C}_{X}\right)$, where $\mathscr{C}_{X}$ is the family of all connected subsets of $X$, together with $X$, and $\mathscr{C}_{Y}$ is any family consisting of the core of $\mathscr{P}(Y)$ together with any collection of connected subsets of $Y$.

Proof. It follows from Theorem (1.9) that both $\left(X, \mathscr{C}_{X}\right)$ and $\left(Y, \mathscr{C}_{Y}\right)$ are $\mathfrak{R}$-spaces. The verification that $\left(Y, \mathscr{C}_{Y}\right)$ is actually a dependant of $\left(X, \mathscr{C}_{X}\right)$ proceeds in much the same way as in the proof of Theorem (1.9). If $Y$ is totally disconnected, $\mathscr{C}_{Y}$ coincides with the core of $\mathscr{P}(Y)$ and the conclusion follows at once. Otherwise, $Y$ contains a nondegenerate connected subset and, for any connected subset $H$ of $Y$, one constructs, just as in the proof of Theorem (1.9), a function $f$ with the properties that $f[X]=f[Y]=H$ and the image of any connected subset of $X$ is either a singleton or all of $H$.

We note that, if $Y$ happens to be connected, then $\mathscr{C}_{Y}$ agrees with $\mathscr{L}_{Y}$. Moreover, in view of Theorem (2.4), we can easily describe all $\mathfrak{R}$-families on $Y$ which result in dependants of $\left(X, \mathscr{C}_{X}\right)$. They are simply all families of the form $\mathscr{C}_{Y}$.

We observed earlier that one cannot always expect to get an $\mathfrak{R}$-family for a subset $Y$ by intersecting $Y$ with the members of an $\mathfrak{R}$-family on $X$. As the next result shows, however, this is a valid procedure for an important class of $\mathfrak{R}$-spaces.

THEOREM (2.5). Let $\left(X, \mathscr{F}_{X}\right)$ be a principal $\mathfrak{R}$-space and let $Y$ be a nonempty subset of $X$. Then $\mathscr{F}_{Y}=\left\{Y \cap H: H \in \mathscr{F}_{X}\right\}$ is a principal $\mathfrak{R}$-family for $Y$ and $\left(Y, \mathscr{F}_{Y}\right)$ is a dependant of $\left(X, \mathscr{F}_{X}\right)$.

Proof. It is immediate that $\mathscr{F}_{Y}$ is a principal $\mathfrak{R}$-family for $Y$. To see that $\left(Y, \mathscr{F}_{Y}\right)$ is a dependant of $\left(X, \mathscr{F}_{X}\right)$, let $H$ be any nonempty set which belongs to $\mathscr{F}_{Y}$. Then $H=K \cap Y$, where $K \in \mathscr{F}_{X}$. Choose $p \in H$ and define a self map $f$ of $X$ by

$$
\begin{array}{lll}
f(x)=x & \text { for } & x \in K, \text { and } \\
f(x)=p & \text { for } & x \in X-K .
\end{array}
$$

It is evident that $f[Y]=H$. Furthermore, for any $V \in \mathscr{F}_{X}$, we have

$$
f[V]=f[V \cap K] \cup f[V-K]=[V \cap K] \cup f[V-K] .
$$

The set $V \cap K$ belongs to $\mathscr{F}_{X}$ and $f[V-K]$ is either empty or consists of the point $p$. In either event, $[V \cap K] \cup f[V-K]$ belongs to $\mathscr{F}_{X}$ and we conclude that $f$ is an $\mathscr{F}_{X}$-invariant function. In the same manner, if $W \in \mathscr{F}_{Y}$, we have

$$
f[W]=[W \cap K\rceil \cup f[Y-K],
$$

and it follows that the restriction of $f$ to $Y$ is an $\mathscr{F}_{Y}$-invariant selfmap of $Y$. 


\section{Restrictive star semigroups.}

Definition (3.1). Let $\left(Y, \mathscr{F}_{Y}\right)$ be a dependant of the $\mathfrak{R}$-space $\left(X, \mathscr{F}_{X}\right)$. Then we denote by $\operatorname{St}(X, Y)$ the semigroup, under composition, of all $\mathscr{F}_{X}$-invariant selfmaps of $X$ whose restrictions to $Y$ are $\mathscr{F}_{Y}$-invariant selfmaps of $Y$. Such semigroups will be referred to as restrictive star semigroups. In the special case where $Y$ is identical with $X$ (from which it necessarily follows that $\mathscr{F}_{Y}=\mathscr{F}_{X}$ ), we use the notation $\operatorname{St}(X)$ and refer to this as a star semigroup.

Our main efforts in this section are directed toward determining precisely when

$$
\operatorname{St}(Z) \text { is a homomorphic image of } \operatorname{St}(X, Y)
$$

and more specifically, when

$$
\text { St }(Z) \text { and } \operatorname{St}(X, Y) \text { are isomorphic. }
$$

As we mentioned in the introduction, this has been done for semigroups of continuous functions in [4], [7] and [8]. As we will see, the answer to (A) is rather similar to that for the analogous problem for semigroups of continuous functions. The answer to (B), however, is very different from its counterpart for semigroups of continuous functions. We assume when proving these results that $\operatorname{St}(X, Y)$ is doubly transitive on $Y$. That is, for each quadruple of points $p, q, r, s$ of $Y$ with $p \neq q$, there exists an $f \in \operatorname{St}(X, Y)$ such that $f(p)=r$ and $f(q)=s$. The next several results indicate that this is not really a serious restriction.

Theorem (3.2). Suppose that both $\mathscr{F}_{X}$ and $\mathscr{F}_{Y}$ are principal $\mathfrak{R}$-families. Then $\operatorname{St}(X, Y)$ is doubly transitive on $Y$.

Proof. This is a consequence of the fact that any selfmap of $X$ whose range is a finite subset of $Y$ is $\mathscr{F}_{X}$-invariant and its restriction to $Y$ is $\mathscr{F}_{Y}$-invariant.

THEOREM (3.3). Let $X$ be a completely regular Hausdorff space with cardinality $c$ and let $Y$ be any nonempty subspace which is not totally disconnected. Let $\mathscr{C}_{X}$ and $\mathscr{C}_{Y}$ be the $\mathfrak{R}$-families on $X$ and $Y$ respectively. Then $\operatorname{St}(X, Y)$ is doubly transitive on $Y$ if and only if $Y$ is connected.

Proof. If $Y$ is connected, one can modify the proof of Theorem (1.9) to produce the desired result. Suppose, on the other hand, that $Y$ is not connected. Then $Y$ is the union of two nonempty disjoint open subsets $A$ and $B$. Since $Y$ is not totally disconnected, one of the two, say $A$, must contain two distinct points $p$ and $q$ which belong to a connected subset of $Y$. Choose any $r \in B$. There exists no function $f$ in $\operatorname{St}(X, Y)$ such that $f(p)=p$ and $f(q)=r$.

As for totally disconnected $Y, \operatorname{St}(X, Y)$ is doubly transitive on some $Y$ but not on others. For example, one readily shows (for any $Y$ ) that if two distinct points of $Y$ belong to the same component in $X$ and a third point of $Y$ belongs to a different component in $X$, then $\operatorname{St}(X, Y)$ is not doubly transitive on $Y$. As the next result shows, however, $\operatorname{St}(X, Y)$ is doubly transitive on many totally disconnected $Y$. 
THEOREM (3.4). Suppose that $X$ is a normal Hausdorff space with cardinality $c$ and $Y$ is a countable metrizable closed subset which is contained in a connected subset of $X$. Again, let $\mathscr{C}_{X}$ and $\mathscr{C}_{Y}$ be the $\Re$-families associated with $X$ and $Y$ respectively. Then $\operatorname{St}(X, Y)$ is doubly transitive on $Y$.

Proof. Since $Y$ is countable, it must be totally disconnected and, consequently, $\mathscr{C}_{Y}$ coincides with the core of $\mathscr{P}(Y)$. Thus the $\mathscr{C}_{Y}$-invariant selfmaps of $Y$ are the constant maps together with all functions which map $Y$ onto $Y$. Now let $p, q, r, s$ be points of $Y$ with $p \neq q$. We prove the existence of a $\mathscr{C}_{X}$-invariant selfmap $f$ of $X$ such that $f(p)=r$ and $f(q)=s$ and the restriction of $f$ to $Y$ is $\mathscr{C}_{Y}$-invariant. We can assume $r \neq s$ since otherwise a constant map will suffice. By Corollary $1[9, \mathrm{p} .141]$, there exists a homeomorphism $g$ from $Y$ into the rational numbers which lie between 0 and 1 . Let $H$ denote a connected subset of $X$ which contains $Y$. Since $H$ has cardinality $c$, there are two distinct points $a, b \in H-Y$. Extend $g$ by defining $g(a)=0$ and $g(b)=1$. Then $g$ maps $Y \cup\{a, b\}$ continuously into the closed unit interval $I$ and, by The Tietze Extension Theorem, can be continuously extended to a function $g^{\prime}$ which maps all of $X$ into $I$. Now we define a function $h$ mapping $I$ into $I$. For rational $x$, let $h(x)=x$. For irrational $x$, let

$$
. a_{1} a_{2} a_{3} \ldots a_{n} \ldots\left(a_{i}=0 \text { or } 1\right)
$$

denote the nonterminating binary expansion of $x$ and let

$$
h(x)=\lim \sup \left\{\left(a_{1}+a_{2}+\ldots+a_{n}\right) / n\right\} .
$$

One can show that the image under $h$ of any nondegenerate interval of $I$ is all of $I$. Finally, let $k$ denote any function mapping $I$ onto $H$ with the properties that

$$
\begin{aligned}
k\left(g^{\prime}(p)\right)=r, & k\left(g^{\prime}(q)\right)=s, \\
k\left(g^{\prime}(r)\right)=p, & k\left(g^{\prime}(s)\right)=q, \\
k\left(g^{\prime}(y)\right)=y & \text { for } \quad y \neq p, q, r, s .
\end{aligned}
$$

Define $f=k \circ h \circ g^{\prime}$. We note that the restriction of $f$ to $Y$ maps $Y$ bijectively onto itself and, consequently, is $\mathscr{C}_{Y}$-invariant. Furthermore, $f(p)=r$ and $f(q)=s$. It remains to show that $f$ is $\mathscr{C}_{X}$-invariant. Let $K$ be any connected subset of $X$. Then $g^{\prime}[K]$ is connected and must be either a point or a nondegenerate subinterval of $I$. In the first case $h\left[g^{\prime}[K]\right]$ is a point and in the second it is all of $I$. It follows that $f[K]$ is either a point or all of $H$.

It is convenient to have two more definitions before we state and prove the two main results of this section.

Definition (3.5). A bijection $h$ from an $\mathfrak{R}$-space $\left(X, \mathscr{F}_{X}\right)$ onto an $\mathfrak{R}$-space $\left(Y, \mathscr{F}_{Y}\right)$ is referred to as an $\mathfrak{R}$-bijection if $h[A] \in \mathscr{F}_{Y}$ for each $A \in \mathscr{F}_{X}$ and $h^{-1}[B] \in \mathscr{F}_{X}$ for each $B \in \mathscr{F}_{Y}$. When such a bijection exists between two $\mathfrak{R}$-spaces, we say that they are equivalent.

DEFINITION (3.6). A dependant $\left(Y, \mathscr{F}_{Y}\right)$ of $\left(X, \mathscr{F}_{X}\right)$ is said to conform to $\left(X, \mathscr{F}_{X}\right)$ if each $\mathscr{F}_{Y}$-invariant selfmap of $Y$ can be extended to an $\mathscr{F}_{X}$-invariant selfmap of $X$. At times, when there can be no misunderstanding as to the $\mathfrak{R}$-families involved, we shall say simply that $Y$ conforms to $X$. 
In the following two theorems, $\left(X, \mathscr{F}_{X}\right),\left(Y, \mathscr{F}_{Y}\right)$ and $\left(Z, \mathscr{F}_{Z}\right)$ are all $\mathfrak{R}$-spaces and $\left(Y, \mathscr{F}_{Y}\right)$ is a dependant of $\left(X, \mathscr{F}_{X}\right)$.

THEOREM (3.7). Suppose that $\operatorname{St}(X, Y)$ is doubly transitive on $Y$ and $Z$ has more than one point. Then $\operatorname{St}(Z)$ is a homomorphic image of $\operatorname{St}(X, Y)$ if and only if $Z$ and $Y$ are equivalent and $Y$ conforms to $X$.

Proof. First suppose that $\varphi$ is a homomorphism from $\operatorname{St}(X, Y)$ onto $\operatorname{St}(Z)$. For each $p \in X$, we let $\langle p\rangle$ denote the function which maps every point of $X$ into $p$, i.e., $\langle p\rangle(x)=p$ for each $x \in X$. It follows that $\{\langle y\rangle: y \in Y\}$ is the kernel (minimal ideal) of $\operatorname{St}(X, Y)$ and $\{\langle z\rangle: z \in Z\}$ is the kernel of $\operatorname{St}(Z)$. Then, for any $y \in Y$, there exists a $z \in Z$ such that $\varphi\langle y\rangle=\langle z\rangle$. We define $h(y)=z$. Then $h$ is a function mapping $Y$ into $Z$ with the property

$$
\varphi\langle y\rangle=\langle h(y)\rangle
$$

for each $y \in Y$.

Now let $z$ be any point of $Z$. Since $\varphi$ is surjective, $\varphi(f)=\langle z\rangle$ for some $f \in \operatorname{St}(X, Y)$. Then, for any $p \in Y$,

$$
\varphi\langle f(p)\rangle=\varphi(f \circ\langle p\rangle)=\varphi(f) \circ \varphi\langle p\rangle=\langle z\rangle \circ \varphi\langle p\rangle=\langle z\rangle .
$$

Thus each $\langle z\rangle$ is the image of at least one element in the kernel of $\operatorname{St}(X, Y)$. Choose any $y \in Y$ such that $\varphi\langle y\rangle=\langle z\rangle$ and define $k(z)=y$. Then $k$ is a mapping from $Z$ into $Y$ such that

$$
\varphi\langle k(z)\rangle=\langle z\rangle
$$

for each $z \in Z$. Now let any $f \in \operatorname{St}(X, Y)$ and any $z \in Z$ be given. Using both (3.7.1) and (3.7.2), we get

$$
\begin{aligned}
& \begin{aligned}
(h \circ f \circ k)(z)=\langle h(f(k(z)))\rangle(z)=\varphi\langle f(k(z))\rangle(z)=\varphi(f \circ\langle k(z)\rangle)(z)=(\varphi(f) \circ \varphi\langle k(z)\rangle)(z) \\
=(\varphi(f) \circ\langle z\rangle)(z)=\varphi(f)(z) .
\end{aligned} \\
& \text { Therefore }
\end{aligned}
$$

$$
\varphi(f)=h \circ f \circ k \text { for each } f \text { in } \operatorname{St}(X, Y) .
$$

Let $i_{X}, i_{Y}, i_{Z}$ denote the identity functions on $X, Y$ and $Z$ respectively. Then $\varphi\left(i_{X}\right)=i_{Z}$ and it follows from (3.7.3) that

$$
h \circ k=i_{z}
$$

Suppose, however, that

$$
k \circ h \neq i_{\mathrm{Y}} .
$$

Then there exists a point $p \in Y$ such that

$$
k(h(p)) \neq p .
$$

Choose any $z \in Z$ and get

$$
(k \circ h \circ\langle p\rangle \circ k)(z) \neq p .
$$


Now assume that $h[Y]$ consists of one point $q$, i.e., $h(y)=q$ for each $y \in Y$. This, together with (3.7.3), implies that $\varphi(f)=\langle q\rangle$ for each $f \in \operatorname{St}(X, Y)$. But the semigroup St $(Z)$ has more than one element since $Z$ has more than one point, and this contradicts the fact that $\varphi$ is surjective. Consequently, we must conclude that there exist points $r$ and $s$ in $Y$ such that $h(r) \neq h(s)$. Since $\operatorname{St}(X, Y)$ is doubly transitive on $Y$, there exists a function $f$ in $\operatorname{St}(X, Y)$ such that

It follows that

$$
f(p)=r \text { and }(f \circ k \circ h \circ\langle p\rangle \circ k)(z)=s .
$$

$$
(h \circ f \circ\langle p\rangle \circ k)(z) \neq(h \circ f \circ k \circ h \circ\langle p\rangle \circ k)(z),
$$

which is a contradiction since $h \circ f \circ\langle p\rangle \circ k$ is $\varphi(f \circ\langle p\rangle)$ and $h \circ f \circ k \circ h \circ\langle p\rangle \circ k$ is $\varphi(f) \circ \varphi\langle p\rangle$. Therefore statement (3.7.5) is not valid and we conclude that

$$
k \circ h=i_{Y} .
$$

Statements (3.7.4) and (3.7.8) together imply that $h$ is a bijection from $Y$ onto $Z$ and that $k=h^{-1}$. Thus (3.7.3) may be rewritten as

$$
\varphi(f)=h \circ f \circ h^{-1} \text { for each } f \in \operatorname{St}(X, Y) .
$$

Next, we show that $h$ is an $\Re$-bijection. For any $H \in \mathscr{F}_{Y}$, there exists a function $f \in \operatorname{St}(X, Y)$ such that $f[Y]=H$. This is a consequence of the fact that $\left(Y, \mathscr{F}_{Y}\right)$ is a dependant of $\left(X, \mathscr{F}_{X}\right)$. Then we have

$$
h\left[H \mathrm{j}=h[f[Y]]=h \circ f \circ h^{-1}[Z]=\varphi(f)[Z],\right.
$$

which implies that $h[H]$ belongs to $\mathscr{F}_{\mathrm{z}}$.

Now suppose that $K \in \mathscr{F}_{Z}$. Let $g$ be any function in St $(Z)$ whose range is $K$ and choose any function $j$ such that $\varphi(j)=g$. By (3.7.9), $g=h \circ j \circ h^{-1}$ and we have

$$
h^{-1}[K]=h^{-1}[g[Z]]=h^{-1}[g[h[Y]]]=j[Y] .
$$

Since $j[Y]$ belongs to $\mathscr{F}_{Y}$, it follows that $h$ is an $\Re$-bijection.

In order to show that $Y$ conforms to $X$, let $f$ be any $\mathscr{F}_{Y}$-invariant selfmap of $Y$. Then $h \circ f \circ h^{-1}$ belongs to St $(Z)$ and, since $\varphi$ is surjective, $\varphi(g)=h \circ f \circ h^{-1}$ for some $g$ in St $(X, Y)$. It follows from (3.7.9) that $h \circ g \circ h^{-1}=h \circ f \circ h^{-1}$. This implies that $g$ is an extension of $f$ and the necessity portion of the proof is complete.

Sufficiency follows quite readily. Suppose that $h$ is an $\Re$-bijection from $Y$ onto $Z$ and that $Y$ conforms to $X$. One easily shows that the mapping $\varphi$ which is defined by

$$
\varphi(f)=h \circ f \circ h^{-1},
$$

for each $f$ in $\operatorname{St}(X, Y)$, is a homomorphism from the latter into St $(Z)$. Let $g$ be any element in $\operatorname{St}(Z)$. Then $h^{-1} \circ g \circ h \in \operatorname{St}(Y)$ and, since $Y$ conforms to $X$, there exists a function $f$ in St $(X, Y)$ whose restriction to $Y$ is $h^{-1} \circ g \circ h$. It follows that $\varphi(f)=g$. Thus $\varphi$ is surjective and the proof is complete.

THEOREM (3.8). Suppose that $\operatorname{St}(X, Y)$ is doubly transitive on $Y$ and also that all two element subsets of $X$ belong to $\mathscr{F}_{X}$. Then $\operatorname{St}(Z)$ and $\operatorname{St}(X, Y)$ are isomorphic if and only if $Z$ and $Y$ are equivalent and $Y=X$. When $Y=X$ it necessarily follows that $\mathscr{F}_{Y}=\mathscr{F}_{X}$. 
Proof. First of all, one easily checks that, if $\left(Y, \mathscr{F}_{Y}\right)$ is a dependant of $\left(X, \mathscr{F}_{X}\right)$ and $Y=X$, then $\mathscr{F}_{Y}=\mathscr{F}_{X}$. The sufficiency portion of the proof is immediate so we comment only on the necessity. It follows quite readily that, if $\operatorname{St}(Z)$ and $\operatorname{St}(X, Y)$ are isomorphic and $Z$ consists of only one point, then $Y$ also consists of one point and $X=Y$. As for the case where $Z$ has more than one point, there exists, just as in the proof of Theorem (3.7), an $\Re$-bijection $h$ from $Y$ onto $Z$ such that

$$
\varphi(f)=h \circ f \circ h^{-1}
$$

for each $f \in \operatorname{St}(X, Y)$, where $\varphi$ denotes an isomorphism from $\operatorname{St}(X, Y)$ onto $\operatorname{St}(Z)$. If $Y \neq X$, choose any $p \in X-Y$ and $q \in Y$ and define

$$
\begin{gathered}
f(x)=p \text { for } x \in X-Y, \\
f(x)=q \text { for } x \in Y .
\end{gathered}
$$

Since all two element subsets of $X$ belong to $\mathscr{F}_{X}$, it follows that $f$ is an $F_{X}$-invariant selfmap of $X$. Moreover, it is immediate that its restriction to $Y$ is an $\mathscr{F}_{Y}$-invariant selfmap. Thus $f$ belongs to $\mathrm{St}(X, Y)$. We have reached a contradiction, since

$$
\varphi(f)=\langle h(q)\rangle=\varphi\langle q\rangle
$$

but $f \neq\langle q\rangle$. We conclude, therefore, that $Y=X$.

Remarks. Theorem (3.7) is very much like its counterpart for semigroups of continuous functions [7, Theorem (4.1) and 8, Theorem (3.2)]. Theorem (3.8), on the other hand, differs in a very important respect from its counterpart in semigroups of continuous functions. Whereas there are some important nontrivial cases where $S(X, Y)$ is isomorphic to a semigroup of the form $S(Z)$ [8, Theorems (4.7) and (4.8)], many of the semigroups $\operatorname{St}(X, Y)$ can only be isomorphic to an $\operatorname{St}(Z)$ if $Y=X$, which then implies that $\mathscr{F}_{Y}=\mathscr{F}_{X}$, i.e., the two $\mathfrak{R}$-spaces $\left(X, \mathscr{F}_{X}\right)$ and $\left(Y, \mathscr{F}_{Y}\right)$ are identical.

Theorem (3.7) emphasizes the need to be able to determine whenever $Y$ conforms to $X$, that is, to be able to determine whenever certain selfmaps of $Y$ all have a certain type of extension to a selfmap of $X$. This is not always an easy task but it is completely determined for semigroups of closed functions on first countable Hausdorff spaces in the next section.

One more remark about Theorem (3.8) seems to be in order. The condition that $\mathscr{F}_{X}$ contain all two element subsets rules out $\mathfrak{R}$-families of the form $\mathscr{C}_{\boldsymbol{X}}$. The following example shows, however, that one cannot hope to prove the theorem without some such restriction.

EXAmple (3.9). Let $X$ be a totally disconnected space with more than one point and let $Y=X-\{p\}$, where $p$ is any point that belongs to $X$. Let $\mathscr{C}_{X}$ and $\mathscr{C}_{Y}$ be the $\mathfrak{R}$-families associated with $X$ and $Y$ respectively. Then $\mathscr{C}_{X}$ is the core of $\mathscr{P}(X), \mathscr{C}_{Y}$ is the core of $\mathscr{P}(Y)$ and $\operatorname{St}(X, Y)$ consists of all constant functions $\langle y\rangle$, where $y \in Y$, together with all those functions which map $X$ onto $X$ and $Y$ onto $Y$. The semigroup St $(Y)$ consists of all constant functions on $Y$ together with all those functions which map $Y$ onto $Y$. One easily verifies that the restriction of each function in $\operatorname{St}(X, Y)$ to $Y$ is an isomorphism from $\operatorname{St}(X, Y)$ onto $\operatorname{St}(Y)$. 
4. Semigroups of closed functions. It will be convenient to assume that all topological spaces discussed in the remainder of this paper are $T_{1}$ spaces. We shall apply some of the previous results to semigroups of closed functions on such spaces. By a closed function, we mean a function with the property that the image of every closed subset is closed.

Let $Y$ be any nonempty subspace of a topological space $X$. By Corollary (1.6), $X$, together with its closed subsets, and $Y$, together with its closed subsets, are $\mathfrak{R}$-spaces. Moreover, it follows from Theorem (2.5) that $Y$ is a dependant of $X$ and it follows from Theorem (3.2) that $\operatorname{St}(X, Y)$ is doubly transitive on $Y$. The semigroup $\operatorname{St}(X, Y)$ in this particular case is the semigroup of all closed selfmaps of $X$ whose restriction to $Y$ is a closed selfmap of $Y$. We shall denote this particular semigroup by $\Gamma^{*}(X, Y)$. If $X=Y$, we have the semigroup of all closed selfmaps of $Y$. We shall denote this semigroup by $\Gamma(Y)$ in keeping with our notation in previous papers. The symbol $\Gamma(X, Y)$ has been used in [6] to denote a semigroup which, although related to $\Gamma^{*}(X, Y)$, may well differ from it. We shall later pursue this further.

It is immediate that, when the $R$-families under consideration are the closed subsets of topological spaces, the $\Re$-bijections are simply homeomorphisms. Theorem (3.8) then translates into

THEOREM (4.1). $\Gamma^{*}(X, Y)$ is isomorphic to $\Gamma(Z)$ if and only if $Z$ is homeomorphic to $Y$ and $Y=X$.

It follows from Theorem (3.7) that, for a space $Z$ with more than one point, $\Gamma(Z)$ is a homomorphic image of $\Gamma^{*}(X, Y)$ if and only if $Z$ is homeomorphic to $Y$ and $Y$ conforms to $X$. In this case, to say that $Y$ conforms to $X$ means that every closed selfmap of $Y$ can be extended to a closed selfmap of $X$. The problem now, of course, is to determine precisely when a subspace $Y$ conforms to $X$ in this manner. The next result determines this completely for first countable Hausdorff spaces; so for such spaces we get a satisfactory solution to the problem of determining when $\Gamma(Z)$ is a homomorphic image of $\Gamma^{*}(X, Y)$. We recall that a space is said to be first countable if each point has a countable basis.

THEOREM (4.2). Let $X$ be a first countable Hausdorff space and let $Y$ be any subspace of $X$. Then each closed selfmap of $Y$ can be extended to a closed selfmap of $X$ if and only if $Y$ satisfies at least one of the two following conditions:

$$
\begin{aligned}
& Y \text { is a closed subset of } X, \\
& Y \text { is discrete and } Y \cup\{t\} \text { is compact for some } t \in X .
\end{aligned}
$$

This latter result, together with Theorem (3.7), yields at once the following

THEOREM (4.3). Let $Y$ be a subspace of a first countable Hausdorff space $X$ and let $Z$ be a topological space with more than one point. Then $\Gamma(Z)$ is a homomorphic image of $\Gamma^{*}(X, Y)$ if and only if $Z$ is homeomorphic to $Y$ and $Y$ satisfies at least one of the two following conditions:

$Y$ is a closed subset of $X$, 
Proof of Theorem (4.2). We first prove necessity. We show that the assumption that $Y$ is not closed leads to the conclusion that $Y$ is discrete and that $Y \cup\{t\}$ is compact for some $t \in X$.

Let us assume that $Y$ is not discrete. Then, since $Y$ is first countable, there exists a sequence $\left\{y_{n}\right\}_{n=1}^{\infty}$ of distinct points of $Y$ converging to some point $q \in Y$. Since $Y$ is not closed, there exists a sequence of distinct points $\left\{x_{n}\right\}_{n=1}^{\infty}$ of $Y$ converging to some point $t \in \mathrm{cl}_{X} Y-Y$ $\left(\operatorname{cl}_{X} Y\right.$ denotes the closure of $Y$ in $\left.X\right)$. Define a function $f$ mapping $Y$ into $Y$ by

and

$$
f\left(y_{n}\right)=x_{n}
$$

$$
f(y)=x_{1} \text { for } y \in Y-\left\{y_{n}\right\}_{n=1}^{\infty} \text {. }
$$

Let $A=\{q\} \cup\left\{y_{n}\right\}_{n=1}^{\infty}$ and $B=\left\{x_{n}\right\}_{n=1}^{\infty}$. Since every subset of $B$ is closed in $Y$, the function $f$ mapping $Y$ into $Y$ is a closed function. Let $k$ be any extension of $f$ which maps $X$ into $X$. Now $A$ is a closed subset of $X$ since $S$ is Hausdorff, but $k[A]=h[A]=B$ is not closed in $X$ since $t \in \mathrm{cl}_{X} B-B$, i.e., $f$ has no extension to a closed function which maps $X$ into $X$. This contradicts the fact that every closed selfmap of $Y$ can be extended to a closed selfmap of $X$; so we must conclude that $Y$ is discrete. We make the observation that, because $Y$ is discrete, every function which maps $Y$ into $Y$ is a closed function.

Now suppose that, in addition to $t, \mathrm{cl}_{X} Y-Y$ contains at least one other point $r$. Then there exists a sequence $\left\{y_{n}\right\}_{n=1}^{\infty}$ of distinct points of $Y$ converging to $r$. Define a function $f$ mapping $Y$ into $Y$ by

and

$$
\begin{gathered}
f\left(x_{2 n-1}\right)=x_{2 n-1}, \\
f\left(x_{2 n}\right)=y_{2 n}
\end{gathered}
$$

$$
f(y)=x_{1} \text { for } y \in Y-\left\{x_{n}\right\}_{n=1}^{\infty} \text {. }
$$

The function $f$ is closed since, as we noted previously, any function mapping $Y$ into $Y$ is a closed function. Let $A=\{t\} \cup\left\{x_{n}\right\}_{n=1}^{\infty}$ and let $k$ be any extension of $f$ which maps $X$ into $X$. Then $A$ is a closed subset of $X$, but $k[A]$ is not a closed subset of $X$ since both $t$ and $r$ belong to $\mathrm{cl}_{X} k[A]$ but at most one of the points can belong to $k[A]$. Specifically, $r \notin k[A]$ if $k(t)=t$, $t \notin k[A]$ if $k(t)=r$ and neither of the points belong to $k[A]$ if $t \neq k(t) \neq r$. Thus, $f$ cannot be extended to a closed function mapping $X$ into $X$. Again we have a contradiction and we conclude that $\mathrm{cl}_{X} Y-Y=\{t\}$.

Now let $H$ be any subset of $Y$ which is closed in $X$. Assume that $H$ is infinite. Then $H$ contains a countable subset $\left\{y_{n}\right\}_{n=1}^{\infty}$ of distinct points which must necessarily be discrete and closed in $X$. Again let $\left\{x_{n}\right\}_{n=1}^{\infty}$ be an infinite sequence of distinct points of $Y$ which converges to $t$. Define a function $f$ mapping $Y$ into $Y$ by

$$
\begin{aligned}
f\left(y_{n}\right)=x_{n} & \text { for each } n, \\
f(y)=x_{1} & \text { for } y \in Y-\left\{y_{n}\right\}_{n=1}^{\infty} .
\end{aligned}
$$

Again, $f$ is a closed function since $Y$ is discrete. Let $k$ be any extension of $f$ which maps $X$ into $X$. Although $H$ is a closed subset of $X, k[H]=f[H]=\left\{x_{n}\right\}_{n=1}^{\infty}$ is not a closed subset of $X$. This is another contradiction. Consequently, any subset of $Y$ which is closed in $X$ is finite. 
Now we are in a position to show that $Y \cup\{t\}$ is compact. Let $\mathscr{F}$ be any family of closed subsets of $Y \cup\{t\}$ which has the finite intersection property. If $t \in \bigcap \mathscr{F}$, we have the desired conclusion. The remaining case is where $t \notin F_{0}$ for some $F_{0} \in \mathscr{F}$. Since $Y \cup\{t\}$ is closed in $X$, $F_{0}$ is closed in $X$ and, by a previous observation, must be finite. Let $F_{0}=\left\{y_{1}, y_{2}, \ldots, y_{N}\right\}$. If, for each $y_{i} \in F_{0}$, there exists a set $F_{i} \in \mathscr{F}$ such that $y_{i} \notin F_{i}$, we obtain the contradiction that $\bigcap\left\{F_{i}\right\}_{i=0}^{N}=\emptyset$. Consequently $y_{i} \in \bigcap \mathscr{F}$ for some $y_{i} \in F_{0}$, which proves that $Y \cup\{t\}$ is compact.

Now we prove sufficiency. Suppose that $Y$ is a closed subset of $X$ and $f$ is a closed function mapping $Y$ into $Y$. Choose any point $q \in Y$ and define a function $k$ mapping $X$ into $X$ by

$$
\begin{aligned}
& k(x)=f(x) \text { for } \quad x \in Y, \\
& k(x)=q \text { for } x \in X-Y .
\end{aligned}
$$

Then, for any closed subset $H$ of $X$,

$$
k[H]=k[[H \cap Y] \cup[H-Y]]=f[H \cap Y] \cup k[H-Y] .
$$

Now $H \cap Y$ is a closed subset of $Y$ and therefore $f[H \cap Y]$ is a closed subset of $Y$. The set $k[H-Y]$ is either empty or consists of the point $q$. In either event, $k[H]$ is a closed subset of $Y$. Since $Y$ is closed in $X, k[H]$ is also closed in $X$. Thus any closed selfmap of a closed subset $Y$ of $X$ can be extended to a closed selfmap of $X$.

Now suppose that $Y$ is discrete and $Y \cup\{t\}$ is compact for some $t \in X$. We may assume that $t \notin Y$, since otherwise the conclusion follows from the previous case. Let $f$ be any function which maps $Y$ into $Y$. Extend $f$ to a function $k$ which maps $Y \cup\{t\}$ into $Y \cup\{t\}$ by defining

and

$$
k(y)=f(y) \text { for } y \in Y
$$

$$
k(t)=t .
$$

Let $H$ be any closed subset of $Y \cup\{t)$. If $H$ is finite, it is evident that $k[H]$ is closed. If $H$ is infinite, $t \in H$ and it follows that $t \in k[H]$. Therefore $k[H]$ is closed since $t$ is the only limit point of $Y \cup\{t\}$. This shows that $k$ is a closed selfmap of $Y \cup\{t\}$ and the conclusion now follows from the last statement of the preceding paragraph.

5. Some further results and concluding remarks. In [5] we proved some results about the semigroup $\Gamma(X, Y)$, which is the semigroup, under composition, of all closed selfmaps of $X$ which also map $Y$ into $Y$. The semigroup $\Gamma^{*}(X, Y)$ is a subsemigroup of $\Gamma(X, Y)$ and may well be a proper subsemigroup since a closed selfmap of $X$ can map $Y$ into $Y$ without its restriction to $Y$ being a closed selfmap of $Y$. The following result tells us precisely when this happens for first countable Hausdorff spaces.

THeORem (5.1). Let $X$ be a first countable Hausdorff space. Then $\Gamma^{*}(X, Y)=\Gamma(X, Y)$ if and only if $Y$ is either discrete or closed.

Proof. One easily verifies that, if $Y$ is either discrete or a closed subset of $X$, then $\Gamma^{*}(X, Y)=\Gamma(X, Y)$. Let $X$ be a first countable Hausdorff space. If $\Gamma^{*}(X, Y)=\Gamma(X, Y)$, 
then $Y$ must be either discrete or closed; for suppose that $Y$ is neither. Then there exists a sequence $\left\{x_{n}\right\}_{n=1}^{\infty}=A$ of distinct points of $Y$ which converges to a point $p \in X-Y$ and a sequence $\left\{y_{n}\right\}_{n=1}^{\infty}$ of distinct points of $Y$ which converges to a point $q \in Y$. Choose any point $r \in Y$ distinct from $q$ and define a function $f$ mapping $X$ into $Y$ by

$$
\begin{aligned}
f(p) & =q, \\
f\left(x_{n}\right) & =y_{n} \text { for each } n, \\
f(x) & =r \text { for } x \in X-[A \cup\{p\}] .
\end{aligned}
$$

In order to see that $f \in \Gamma(X, Y)$, let $H$ be any closed subset of $X$. If $H \cap A$ is finite, then $f[H]$ is finite and, consequently, is closed. If $H \cap A$ is infinite, then $p \in H$ and $f[H]=\{q\} \cup B$, where $B \subset\{r\} \cup\left\{y_{n}\right\}_{n=1}^{\infty}$. In this case also, $f[H]$ is closed. Thus $f \in \Gamma(X, Y)$. However, the restriction of $f$ to $Y$ is not a closed function from $Y$ into $Y$, because $A$ is a closed subset of $Y$ but $f[A]$ is not. This completes the proof.

It follows from this latter result and Theorem (4.3) that, if $X$ is a first countable Hausdorff space and $\Gamma^{*}(X, Y)$ is a proper subsemigroup of $\Gamma(X, Y)$, then the only homomorphic image of $\Gamma^{*}(X, Y)$ of the form $\Gamma(Z)$ is the one-element semigroup, that is, the case where $Z$ consists of one point.

The main result of [6] is that, if $Y$ contains more than one point and $\varphi$ is any bijection from $\Gamma(X, Y)$ onto $\Gamma(U, V)$, then $\varphi$ is an isomorphism if and only if there exists a homeomorphism from $X$ onto $U$ which also carries $Y$ onto $V$, such that $\varphi(f)=h \circ f \circ h^{-1}$ for each $f$ in $\Gamma(X, Y)$. The techniques used in proving that result will not carry over to semigroups of the form $\operatorname{St}(X, Y)$, where $X$ is any $\mathfrak{R}$-space and $Y$ is a dependant of $X$. The reason for this is that in [6] we repeatedly used the fact that certain nonconstant functions with finite ranges belonged to the semigroups under consideration. This certainly need not be the case for arbitrary St $(X, Y)$. However, the arguments of [6] will carry over in their entirety to semigroups of the form $\operatorname{St}(X, Y)$ when $\left(X, \mathscr{F}_{X}\right)$ is a principal $\mathfrak{R}$-space and $\mathscr{F}_{Y}$ consists of all intersections of $Y$ with members of $\mathscr{F}_{X}$. We state this result formally, without proof:

TheOREm (5.2). Let $\left(X, \mathscr{F}_{X}\right)$ and $\left(U, \mathscr{F}_{U}\right)$ be principal $\mathfrak{R}$-spaces and, for $Y \subset X$ and $V \subset U$, let $\mathscr{F}_{Y}=\left\{Y \cap H: H \in \mathscr{F}_{X}\right\}$ and $\mathscr{F}_{V}=\left\{V \cap H: H \in \mathscr{F}_{U}\right\}$ be the $\mathfrak{R}$-families associated with $Y$ and $V$ respectively. Assume further that $Y$ and $V$ have more than one point. Then $a$ bijection $\varphi$ from $\operatorname{St}(X, Y)$ onto $\operatorname{St}(U, V)$ is an isomorphism if and only if there exists an $\mathfrak{R}$-bijection $h$ from $X$ onto $U$ whose restriction to $Y$ is an $\Re$-bijection from $Y$ onto $V$, such that $\varphi(f)=h \circ f \circ h^{-1}$ for each $f$ in $\operatorname{St}(X, Y)$.

The following corollary is a special case of Theorem (5.2).

COROLlaRY (5.3). Let $Y$ and $V$ be subspaces (with more than one point) of the topological spaces $X$ and $U$ respectively. Then a bijection $\varphi$ from $\Gamma^{*}(X, Y)$ onto $\Gamma^{*}(U, V)$ is an isomorphism if and only if there exists a homeomorphism $h$ from $X$ onto $U$ which carries $Y$ onto $V$, such that $\varphi(f)=h \circ f \circ h^{-1}$ for each $f$ in $\Gamma^{*}(X, Y)$.

We conclude by mentioning that it would be interesting to characterize those dependants $\left(Y, \mathscr{F}_{Y}\right)$ of $\left(X, \mathscr{F}_{X}\right)$ which conform to $\left(X, \mathscr{F}_{X}\right)$ for classes of $\Re$-spaces in addition to the $\mathfrak{R}$-spaces 
of closed subsets of first countable Hausdorff spaces. In particular, it would be interesting to know precisely when $\left(Y, \mathscr{C}_{Y}\right)$ conforms to $\left(X, \mathscr{C}_{X}\right)$, that is, for what subsets $Y$ of $X$ can all connected selfmaps of $Y$ with connected range be extended to a connected selfmap of $X$ with connected range (a connected function is one which takes connected sets into connected sets). The answer would result in the counterpart for semigroups of connected functions of Theorem (4.3).

\section{REFERENCES}

1. A. H. Clifford and G. B. Preston, The algebraic theory of semigroups, Amer. Math. Soc. Mathematical Surveys 7, Vols I and II (Providence, R.I., 1961 and 1967).

2. C. Kuratowski, Topologie II (Warsaw, 1950).

3. E. S. Ljapin, Semigroups, Amer. Math. Soc. Translations of Mathematical Monographs 3 (Providence, R.I., 1963).

4. K. D. Magill, Jr., Subsemigroups of $S(X)$, Math. Japon. 11 (1966), 109-115.

5. K. D. Magill, Jr., Semigroup structures for families of functions, III; $\mathfrak{R} r^{*}$-semigroups, J. Australian Math. Soc. 14, part 4 (1967), 524-538. 1215-1229.

6. K. D. Magill, Jr., Restrictive semigroups of closed functions, Canad. J. Math., 20 (1968),

7. K. D. Magill, Jr., Homomorphic images of certain semigroups of continuous functions, Math. Japon. 13 (1968), 133-141.

8. K. D. Magill, Jr., Homomorphisms and semigroups of continuous functions, Proc. Topology Conference (Kanpur, India, October 1968); to appear.

9. W. Sierpinski, General topology (Toronto, 1952).

State University of New York at Buffalo

and

UNIVERSITY OF LEEDS 